\title{
Acute Hemorrhagic Encephalitis
}

National Cancer Institute

\section{Source}

National Cancer Institute. Acute Hemorrhagic Encephalitis. NCI Thesaurus. Code C35796.

Acute encephalitis that is characterized by bleeding. 